\title{
Susceptibility to the effects of delayed auditory feedback
}

\author{
PETER HOWELL \\ University College London, London, England \\ and \\ ALEXANDRA ARCHER \\ National Hospital College of Speech Sciences, London, England
}

\begin{abstract}
Speech performance deteriorates if a delay is introduced between the time when speakers speak a sound and when they hear it (DAF). Most disturbance occurs when speech is delayed by about $200 \mathrm{msec}$. Not all speakers are equally susceptible to the effects of DAF. The hypothesis examined here is that deterioration in performing a serially organized behavior (such as speech) occurs when a rhythmic event goes on concurrent with but out of synchrony with the activity. Different susceptibility to the effects of DAF can be accounted for by this hypothesis because the out-of-synchrony signal would differ in loudness, depending on the amount by which speakers raise their voices under DAF. Two experiments are reported which are consistent with this hypothesis. In Experiment 1 , it is shown that changes in voice level brought about by voluntary or electronic means increase the disturbance observed. In Experiment 2, it is shown that the out-of-synchrony signal does not have to be speech to produce problems for speech control, because equivalent disruption occurs when a nonspeech signal at the same level as the speech is substituted for the delayed speech sound.
\end{abstract}

When auditory feedback of speech is delayed (DAF), speech control suffers. The effects of delaying feedback of speech are that the level and pitch of the voice go up, speech rate goes down, and the speaker makes all types of speech errors (Fairbanks, 1955). These disturbances do not increase monotonically as the delay is increased; speech is most disrupted and error rate is highest at a delay of about $200 \mathrm{msec}$, and voice level and pitch asymptote at about this same delay. The effects of delay on speech and error rate have been explained by assuming that the speaker continues to use the feedback for control when it is not in its normal temporal relationship to speech activity.

For example, the high error rate and slow rate of speech were thought to occur because speakers used the delayed signal to check that they had articulated the sound correctly (Black, 1951). If speech was controlled by instructions issued for a sequence of segments, maximum disruption would occur at a delay that corresponded to the duration of the segments. At this delay, the speaker would think that the feedback he or she was receiving was from the current segment when, in fact, it was from the one he or she had just produced. It follows from this account that the size of the segment involved in speech production is similar in duration to the delay that produces maximum

This research was supported by the Medical Research Council of the United Kingdom. Address reprint requests to Peter Howell, Department of Psychology, University College London, Gower St., London WC1E 6BT, England. disruption. Since speech is most disrupted at a delay of about $200 \mathrm{msec}$ and syllables are about $200 \mathrm{msec}$ long, syllables might be the unit that is used to control speech production.

There are marked differences in the amount of disturbance experienced when speaking under DAF. The experiments reported in this article were directed at ascertaining what determines the susceptibility of different speakers to the effects of DAF. Previous such research has focused on two questions: Can the speakers who are most susceptible and those who are least susceptible be identified on the basis of personality factors, and do groups with different language facilities differ in susceptibility?

The factors that have been examined as candidate predictors of DAF susceptibility include personality variables (Korrowbrow, 1955; Rankin \& Balfrey, 1966), normal speech performance (Butler \& Galloway, 1957), and differences between subjects with respect to whether they rely on auditory or oral sensory feedback (Yates, 1963). Such studies have not succeeded in revealing factors that predict DAF susceptibility. For example, Rankin and Balfrey dismissed anxiety as a personality variable that could account for DAF susceptibility because an anxiety questionnaire did not distinguish speakers who were highly susceptible from those who were not. Butler and Galloway (1957) found no difference in susceptibility between subjects who spoke slowly, at medium speed, or fast. Burke (1975) found that subjects who were highly susceptible to the effects of DAF were affected by alterations to auditory or oral sensory feedback in the same way as subjects who showed 
low susceptibility. Thus, susceptibility to the effects of DAF is not due to differences in subjects' reliance on these sources of feedback (Yates, 1963).

With regard to fluency, the main variables that have been examined are sex of the speaker (Bachrach, 1964), age of the speaker (Siegel, Fehst, Garber, \& Pick, 1980), and whether the speaker is speaking his or her native language or one that was acquired (or learned) later in life (MacKay, 1970). The less fluent the speakers are (i.e., if they are male, young, or using a language other than their first), the more they are affected when speech is delayed. Thus, fluent speakers may rely less on auditory feedback than less fluent speakers.

Although, under DAF, the disturbance of speech may be interpreted in terms of an auditory-feedback regulation account, another account has been offered: Howell, Powell, and Khan (1983) suggested that the effects of DAF arose from interference, in the performance of a serially organized behavior (such as speech), produced by a rhythmic event going on at the same time but out of synchrony with the activity. They assumed that events that occurred at the offset of each serially organized unit were particularly disruptive. To support this assumption they cited, among other things, the difficulty experienced by musicians playing a sequence of notes when other musicians played notes synchronized to the offsets. The sound another musician produces cannot be used as feedback, and yet it causes disruption to performance.

This hypothesis can be applied to DAF of speech as follows. The intensity contour of speech rises and falls periodically, rising at the start of a syllable and dropping at the end. Since syllables are about $200 \mathrm{msec}$ long, when the speech is delayed by $200 \mathrm{msec}$ the intensity contour of the delayed speech should be starting to rise as the intensity contour of the speech currently being produced decreases. ${ }^{1}$ Thus, delaying speech by about $200 \mathrm{msec}$ creates a situation like the one Howell et al. (1983) argued was particularly disruptive in the performance of a task involving serial organization of activity, and this could account for why most disruption occurs at a delay of $200 \mathrm{msec}$ in DAF of speech. This differs from the account of the effects of DAF described earlier, where it was assumed that the delayed signal was used to ascertain whether the sound had been articulated correctly.

With respect to the Howell et al (1983) hypothesis, the question arises as to what determines susceptibility to DAF if the delayed signal is not used for the purposes of feedback control? Howell et al. argued that the louder the delayed signal (or, more generally, any signal that was out of synchrony with speech), the more intrusive it would be and, therefore, the more disruption it should cause. In line with this prediction, Butler and Galloway (1957) have shown that the effects of DAF increase when the level of the delayed signal is amplified, with most increase in disruption occurring at a 200-msec delay. The work referred to so far has been concerned with amplification external to the speaker, and may seem irrelevant to the problem of explaining differential sensitivity to DAF. However, the fact that, because of differences in the level of their voices, intensity of feedback would vary across speakers might be one factor that causes the differences in disruption observed under DAF. Also, speakers might differ in the way in which they alter their voice levels under DAF. In both cases, the level of the intruding event would be increased when voice level was high, causing more disruption to performance.

Some evidence that those speakers who raise their voice levels considerably under DAF experience more disruption than those who raise their voice levels only slightly has been reported by Howell et al. (1983). In their Experiment 2, performance time (PT) and sound pressure level were measured at different delays. They found a positive correlation between the amount speakers raised their voices and the resulting increase in PT.

Other accounts of performance under DAF dismiss level as being of only secondary importance (e.g., Fairbanks, 1955). In the current account, on the other hand, DAF susceptibility derives directly from the level of the delayed signal. This account implies that since speakers can voluntarily alter their voice levels, susceptibility to DAF should not be fixed. This would not be so if, for example, personality factors determined susceptibility to DAF. Howell et al.'s account of DAF disruption predicts that the level of any signal (not just speech) that occurs where the delayed speech would would cause the same disruption. In traditional accounts, on the other hand, it would not be possible to use nonspeech feedback as a substitute for speech for articulatory control so nonspeech should cause less disruption to speech control.

In the experiments reported in this article, we tested whether level of the delayed signal predicts susceptibility to DAF. In the first experiment, performance under DAF was examined with speakers speaking in a normal or a raised voice. If voice level determines susceptibility to DAF, subjects should experience more disruption when they are instructed to increase voice level. In the second experiment, we examined whether a nonspeech sound substituted for the delayed speech signal would be equally as disruptive as speech at the same level.

In Experiment 1, the effects of instructing subjects as to what voice level to employ under DAF were compared with electronic changes, in the manner of Butler and Galloway (1957). PT was measured using a randomized sequence of two vowels. The task was simple enough for subjects not to make errors (Howell et al., 1983). When the feedback was amplified electronically, a peak in the disturbance function (i.e., the function relating PT to delay) was expected at about the same delay that Butler and Galloway (1957) found to produce maximum disruption with errors (i.e., at about $200 \mathrm{msec}$ ). Also, it was expected that the peak should increase as feedback was amplified electronically (Butler \& Galloway, 1957). The effects on PT were expected to be similar to those found by Butler and Galloway on errors, since PT and errors are directly related (Fairbanks, 1955). A second intention was to ascertain whether voluntary changes in speech level produced 
effects similar to those expected with electronic amplification (i.e., to peak at about a 200-msec delay and to increase peakiness as voice level went up). In the conditions in which speakers were instructed to raise voice level, sound pressure level was measured to check the rise in voice level and to see what relationship, if any, existed between the amount by which subjects increased voice level under DAF and the amount of disruption they experienced. The hypothesis derived from Howell et al. (1983) predicted a positive correlation.

\section{EXPERIMENT 1}

\section{Method}

Subjects. Ten females served as subjects in the experiment. Stimulus material. Fifteen lists of randomly ordered $/ \mathrm{i} /$ and $/ \mathrm{u} /$ vowels were used. There were $\mathbf{4 0}$ vowels in each list and an equal number of each of the vowels.

Procedure and Apparatus. The experiment took place in a soundtreated room. A Beyerdynamic microphone was held at a distance of about $20 \mathrm{~cm}$ from her mouth. Oral output was delayed by a PDP-11/10 computer, which was programmed to operate as a variable delay line. Speech was digitized at a $10-\mathrm{kHz}$ rate (after low-pass filtering at $3.5 \mathrm{kHz}$, $48 \mathrm{~dB} /$ octave) and output from the computer (also at $10 \mathrm{kHz}$ ) after the specified delay. The output was also low-pass filtered at $3.5 \mathrm{kHz}$ with a roll-off of $48 \mathrm{~dB} /$ octave, and the speech was relayed to the speaker via headphones (AKG K120R). The subjects had to speak a typed list of the 40 vowels, and feedback was delayed by $0,100,200,300$, and 400 msec.

Each subject participated in both the electronic amplification conditions and the conditions in which she voluntarily raised her own voice level. Electronic amplification was achieved using a Revox. Production was amplified by 10,20 , and $30 \mathrm{~dB}$. The calibration settings were obtained by referencing amplification to a noise source with a known output level (Ivie IE $20 \mathrm{~B}$ ). In the conditions in which the subjects were instructed on how to control voice level, the setting on the amplifier was left at the 10-dB amplification setting used in the electronic amplification conditions and the subject was asked to speak at normal voice level or to raise the level of her voice.

The order in which the subjects received the amplification conditions was counterbalanced across subjects. Order of amplification level and delay were counterbalanced within and across subjects using Latin squares. The subjects were timed by stopwatch. If an error was detected, the list was repeated immediately. In the conditions in which the subjects were instructed on what voice level to employ, the maximum sound pressure level a subject produced while reading the list was recorded. Sound pressure level was measured by a Peters SM6 sound-level meter with its pickup microphone held in a stand located $20 \mathrm{~cm}$ from the speaker's lips. ${ }^{2}$

\section{Results}

The data from the electronic amplification conditions were examined to ascertain (1) whether a peak occurred at a delay of about $200 \mathrm{msec}$, and (2) whether the peakiness increased with amplification. The mean PTs across subjects at each delay and in each of the amplification conditions are presented in Figure 1.

The functions in all three amplification conditions have a peak, and analysis of variance (ANOVA) was used to establish whether this was significant. A repeated meaures ANOVA was performed with two within-subjects variables (level of the signal and delay). The PTs over delays differed significantly $[F(4,36)=11.8, p<.001]$, and the difference over delays involved a quadratic trend $[F(1,9)$ $=10.6, \mathrm{p}<.01]$. The quadratic component across delays

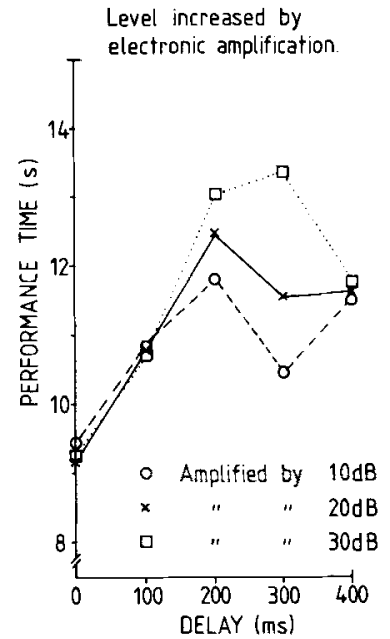

Figure 1. Performance time at different delays for the electronic amplification conditions of Experiment 1. The parameter of the connected points is amplification level (given by the symbol in the inset).

showed that the functions were peaked. The peaks, estimated by polynomial regression analysis, occurred at delays of 300,260 , and $270 \mathrm{msec}$ for the 10-, 20-, and 30-dB amplification conditions, respectively.

Do the functions become more peaked as amplification is increased? If so, the quadratic component of the delay effect should become more marked as amplification level increased, so in the ANOVA should interact with level. The quadratic component of delay interacted with the linear component of level $[\mathrm{F}(1,9)=5.4, \mathrm{p}<.05]$, which showed that the peakiness of the functions was more marked when amplification was high. [The overall interaction between delay and amplification level was significant, too- $F(8,72)=4.5, \mathrm{p}<.001]$. The other significant factor in the analysis was the main effect of level $[F(2,18)$ $=7.3, \mathrm{p}<.01]$, which showed that PTs were longer when amplification was high. In sum, the peak in PT at delays of between 200 and $300 \mathrm{msec}$ and the increase in peakiness as amplification was increased replicate previous findings that disruption is increased when delayed feedback is amplified electronically (Butler \& Galloway, 1957).

To ascertain whether the functions become peakier when an increase in the level of feedback is brought about by the speaker's speaking more loudly, it is first necessary to establish whether subjects increased their voice levels when instructed to do so. The mean level across subjects in the self-regulated voice-level conditions is presented in Figure 2. It appears from this figure that subjects followed the instructions, since mean levels are greatest in the conditions in which the speakers were instructed to raise their voices. This was confirmed by a repeated measures ANOVA with two factors (the level at which speakers were instructed to speak and delay). In this analysis, voice levels were significantly higher in the conditions in which subjects were instructed to increase the level $[F(1,9)=$ $46.9, \mathrm{p}<.001]$, which indicated that the subjects had followed the instructions. The difference in levels across delays was also significant $[F(4,36)=3.6, p<.025]$. 


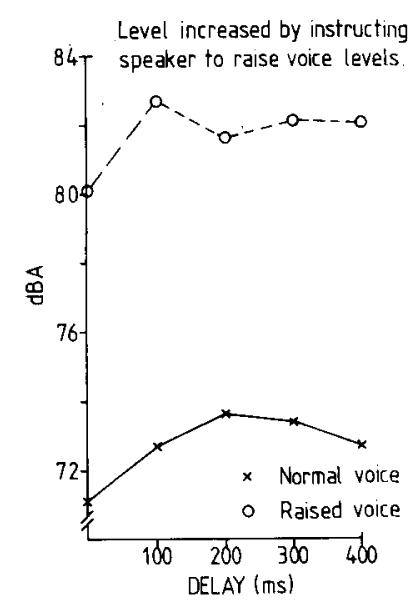

Figure 2. Sound pressure level at different delays for the conditions in which subjects altered their voice levels. The conditions in which the subjects used normal or raised voice are identified by the symbol (given in the inset).

Having established that subjects had increased their voice levels when told to do so, did this result in the functions of PT on delay being more peaked when voice level was increased? The mean PTs obtained are presented in Figure 3, which shows that a bigger peak occurred in the conditions in which the speakers were instructed to raise their voices. These data were analyzed in the same way as in the electronic amplification conditions. The results of the ANOVA paralleled those obtained in the electronic amplification conditions discussed above: the quadratic component of the delay effect was significant, which showed that the functions were peaked $[\mathrm{F}(1,9)=23.3$, $\mathrm{p}$ $<.001]$. The peaks, estimated by polynomial regression, occurred at delays of 300 and $275 \mathrm{msec}$ in the normal and raised-voice conditions, respectively. The linear compo-

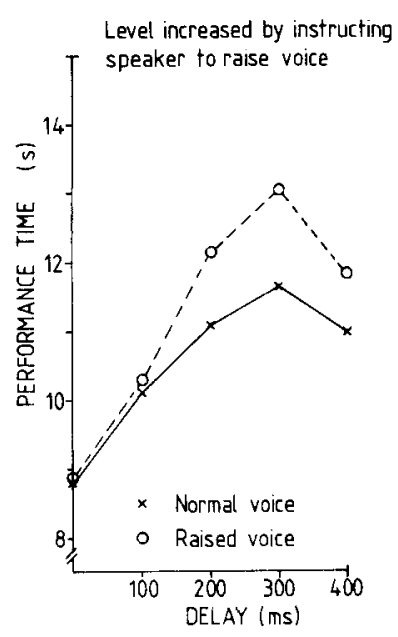

Figure 3. Performance time at different delays for the conditions in which subjects altered their voice levels. The conditions in which the subjects used normal or raised voice are identified by the symbol (given in the inset). nent of the level effect interacted with the quadratic component of the delay effect $[F(1,9)=6.5, p<.05]$, showing that the peak was bigger in the conditions in which speakers raised their voices [the interaction between level and delay was significant too- $\mathrm{F}(4,36)=3.5, \mathrm{p}<.05]$. Also, the main effect of level was significant $[\mathrm{F}(1,9)=$ $7.5, \mathrm{p}<.05]$, with the longest PTs in the raised-voicelevel conditions. In sum, the effect of raising voice level, both electronically or by the speaker's voluntarily adjusting her voice level, caused the disturbance functions to become more peaked.

As discussed in the introduction, Howell et al. (1983) reported that the increase in voice level was correlated with the amount of disruption subjects experience under DAF. A related question is whether the disturbance experienced by subjects was more marked with those subjects who increased their voice levels most in the raised-voice-level condition than with those subjects who raised their voice levels least. To examine whether this was so, the difference between maximum PT and minimum PT (i.e., an index of the amount by which the subject raised her voice) was calculated for the self-regulated voice-level conditions as an index of disruption. A Pearson product moment correlation was performed between this index and the difference between maximum and minimum voice level (i.e., an index of the amount by which speakers raised their voices). The correlation was +.583 , which is significant $(<5 \%)$ on a one-tail test $(\mathrm{df}=8)$. Thus, those subjects who raised their voices more experienced more disruption. As in corresponding analyses performed by Howell et al. (1983) on electronically amplified delayed feedback, there was no correlation between absolute sound pressure level and PT. It appears that, in these experiments, absolute voice level does not determine DAF susceptibility. However, in both of these experiments, the subjects were quite homogeneous in the sound pressure levels they produced.

\section{Discussion}

Similar disruption was observed regardless of whether level changes were achieved by electronic means or by instructing the subjects to raise their voice levels. In both cases, the louder the signal, the more disruptive it was. Also, most disruption was experienced by those subjects who raised their voices most. The finding that disruption caused by DAF is determined by something under voluntary control indicates that susceptibility is not a fixed propensity of the individual.

A question that remains is why different subjects control their voice levels in different ways under DAF. Those speakers who raise their voices under DAF probably do so to increase the amount of direct sound that leaks around the headset and that is transmitted by bone conduction. Such subjects experience considerable disruption because they raise not only the level of direct feedback, but also the level of the delayed feedback. If speakers adopted a different strategy (e.g., not raising voice level), the disruption caused by the delayed signal would not increase as much across delays. This can be appreciated if the ar- 
gument is taken to its limit; if speakers reduced their voice levels so that no sound issued, they would experience no differential effect on PT across delays (i.e., they would show no susceptibility to the effects of DAF at all). Thus, susceptibility may be determined by what strategy of voicelevel control the speaker adopts, and there is nothing inherent about how different speakers use feedback.

The present results do not establish whether the delayed signal is used for feedback control or not. It could be argued that the delayed signal is used to determine whether the sound was articulated correctly and that increasing the level of the delayed signal makes it more difficult to ignore. The variation in susceptibility when voice level is altered would still indicate that although subjects do not have a fixed propensity for the way in which they use feedback, the delayed speech might still be used to regulate the voice.

In the second experiment, we examined the question of whether the delayed signal is used for auditory-feedback regulation of the voice. A comparison is made between the effects of delaying and altering electronically the level of speech feedback and similar manipulations in situations in which a nonspeech signal is substituted for the delayed speech signal. A nonspeech sound was substituted for the delayed speech by first calculating the intensity envelope of the original speech. The intensity envelope can be delayed and used to gate on a nonspeech sound (a square wave in our experiment). The nonspeech sound with the same intensity envelope as the original speech would then be heard after the specified delay. If the disturbance caused by DAF was due to the speaker's continuing to use the delayed speech signal to determine whether the sound was being articulated correctly, more disturbance should occur in that case than with delayed speech feedback. If no difference between speech and nonspeech feedback occurred, it would indicate that the disturbance did not arise because the speaker continued to use the delayed speech for articulatory control. The level of feedback was manipulated in the speech and nonspeech tasks to see whether nonspeech showed effects similar to those of speech when its level is altered (i.e., a bigger peak at a delay of around 200 msec-Experiment 1).

\section{EXPERIMENT 2}

\section{Method}

Subjects. Ten female subjects were used in the experiment. They were taken from the same population as in Experiment 1.

Stimulus material. Stimulus materials were the same as those used in Experiment 1.

Procedure and Apparatus. There were two types of experimental conditions: delayed speech feedback and delayed nonspeech feedback. The procedure for the delayed speech feedback condition was the same as that for the electronic amplification conditions of Experiment 1 . In this condition, in addition to PT, voice level was measured as in the selfregulated voice level conditions of Experiment 1. Amplitude settings and delay were varied as in Experiment 1, and the order subjects received these was counterbalanced across subjects by Latin squares. The pickup microphone of the sound-pressure-level meter was again held in a stand at a constant distance of $20 \mathrm{~cm}$ from the speaker's lips.
In the nonspeech feedback conditions, the experiment proceeded as for the speech-feedback conditions, except that a nonspeech sound was substituted for the speech. This was achieved as follows: The airborne part of speech was picked up by the Beyerdynamic microphone and delayed by the PDP-11/10 computer, as before. The envelope of the delayed speech was isolated using a Fonema speech signal rectifier (linear). This envelope was then fed through a precision linear multiplier (Burr-Brown $4213 \mathrm{BM}$ ), where it was multiplied with a $500-\mathrm{Hz}$ square wave. The result of this arrangement was that the amplitude contour of the original speech gated the square wave on and off. The output of the multiplier was amplified by a Revox and the levels were equated across speech and nonspeech conditions by balancing the outputs for a standard input signal. Level and delay order were received in random order, as with the speech feedback. The order in which subjects received speech or nonspeech feedback was counterbalanced across subjects. Levels were measured as in the speech feedback condition.

\section{Results}

The sound pressure levels for the speech and squarewave conditions are shown in Figure 4. An ANOVA of the sound pressure levels with the within-subjects factors of feedback condition (speech or square wave), level, and delay was performed. The voice levels differed across delays $[F(2,18)=24.8, p<.001]$, as has been reported elsewhere (e.g., Fairbanks, 1955; Howell et al., 1983). Voice level increased with amplification $[F(4,36)=18.3, p<.001]$, again as reported elsewhere (Howell et al., 1983). The ANOVA of the sound pressure levels showed that there was a significant 2.5-dB difference across the speech and nonspeech conditions, with subjects producing the higher sound pressure levels in the nonspeech conditions $[F(1,9)$ $=140.6, \mathrm{p}<.001]$. It is not apparent why there was a

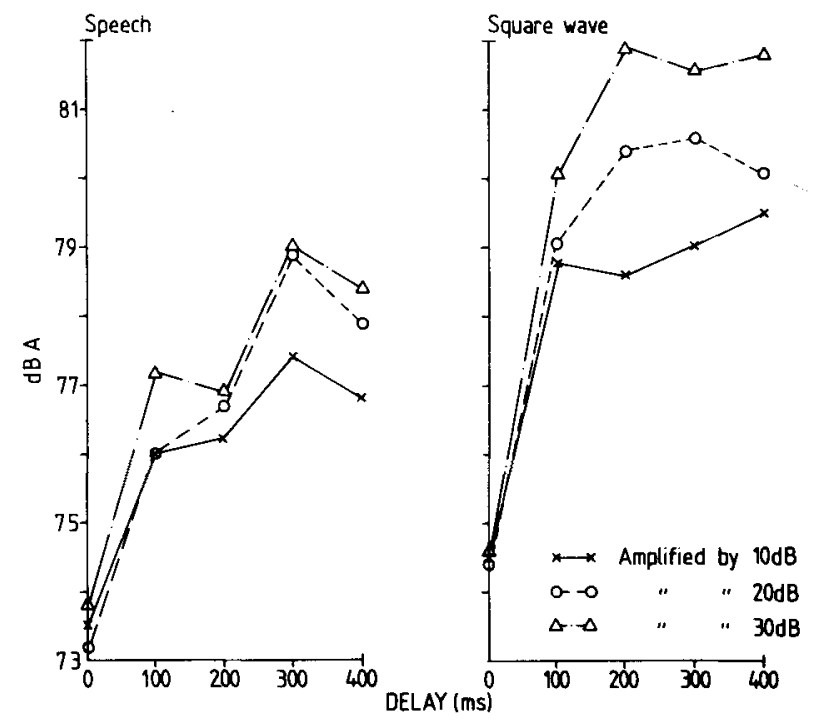

Figure 4. Sound pressure level at different delays for the speech and nonspeech conditions of Experiment 2. The section on the left (labeled "speech") comes from the conditions in which speech was delayed. The section on the right (labeled "square wave") comes from conditions in which a nonspeech sound (a square wave) was substituted for the delayed speech. The parameter of the connected points is from the different amplification conditions and can be identified by the symbol (given in the inset). 
difference between the voice levels used by the speakers in the two feedback conditions, but it necessitated correcting the PTs across the conditions, because otherwise differences between the speech and nonspeech conditions could be attributable to the different voice levels (Experiment 1). What the PT would have been if the speakers had used comparable levels was calculated as follows: At each delay, the PT and level were taken for the square-wave condition. PT was then regressed on level, subject by subject. The regression equation of PT was then used to obtain the PT at the levels used by that speaker in the three speech conditions. The obtained PTs for equivalent levels in the speech and nonspeech conditions are shown in Figure 5 (the uncorrected PTs from the square-wave condition are shown in Figure 6).

An ANOVA of the PTs (using the PTs corrected for the level differences across the speech and nonspeech conditions) with the factors of feedback condition (speech or nonspeech), feedback level, and delay was performed, as with the PTs of the electronic amplification conditions of Experiment 1 . There was no difference between feedback conditions or any interaction between feedback conditions and the other factors. This shows that the disruption caused by a delayed nonspeech signal is equivalent to that with a delayed speech signal of the same level. Thus, as argued in the introduction to this experiment, the delayed speech signal is not used for the purpose of regulatory control of the voice, since nonspeech caused equivalent disruption to speech.

The results of the ANOVA were next examined to ascertain whether the disturbance functions were peaked and

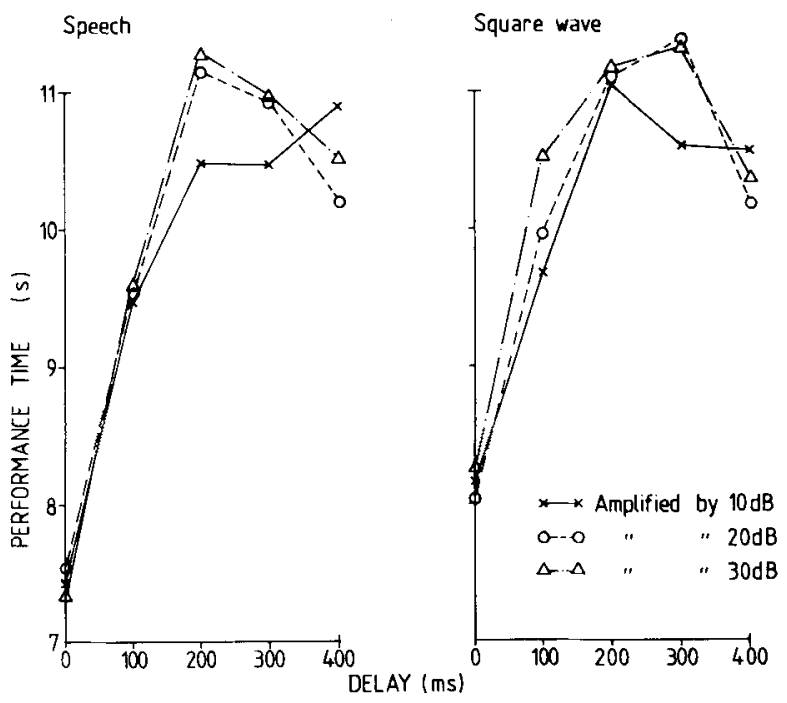

Figure 5. Performance time at different delays for the speech and nonspeech conditions of Experiment 2. The section on the left (labeled "speech") comes from the conditions in which speech was delayed. The section on the right (labeled "square wave") comes from conditions in which a nonspeech sound (a square wave) was substituted for the delayed speech. The parameter of the connected points is from the different amplification conditions and can be identified by the symbol (given in the inset).

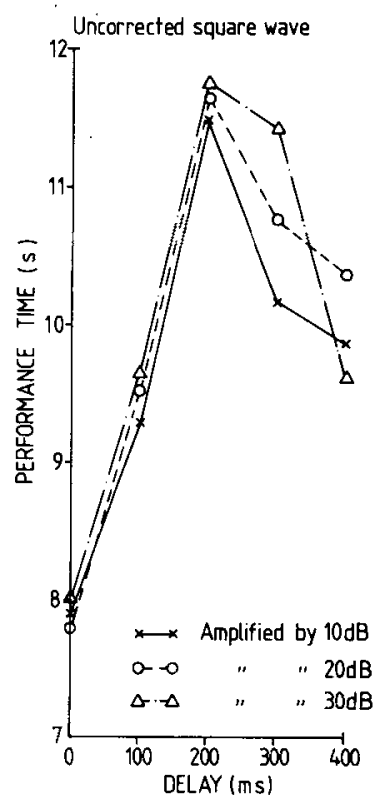

Figure 6. Uncorrected performance times for the nonspeech condition of Experiment 2. The results are presented in the same way as the corresponding results in Figure 5.

whether the peakiness increased with increased amplification, as found in Experiment 1. The PTs differed across delays $[F(4,36)=14.3, p<.001]$, and orthogonal breakdown of the delay effect showed that the quadratic component was significant $[F(1,9)=14.6, p<.01]$. Thus, the functions are peaked and the peaks occurred at delays of 310,265 , and $260 \mathrm{msec}$ for the 10-, 20-, and 30-dB amplification conditions with speech feedback and at 275, 285, and $280 \mathrm{msec}$ for the corresponding conditions with nonspeech feedback (estimated by polynomial regression). The level $\times$ delay interaction showed that the linear component of the delay effect interacted with the quadratic component of level $[F(1,9)=5.3, p<.05]$, which indicated that the size of the peak increased as level was increased (as found in Experiment 1). The level $\times$ delay interaction was significant $[\mathrm{F}(8,72)=2.4, \mathrm{p}<.05]$. A significant effect of feedback level occurred $[F(2,18)=$ $4.3, \mathrm{p}<.05]$, and this arose because PTs increased as level was raised from 10 through 20 to $30 \mathrm{~dB}$ (cf. Butler \& Galloway, 1957; Howell et al., 1983; and Experiment 1 above).

In sum, the results show that performance with delayed speech was similar to performance with nonspeech when the level of the feedback was the same. In both conditions, the disturbance functions across delay were peaked and the peakiness of the disturbance functions increased with level of the feedback.

\section{Discussion}

The results of the present experiment show that performance in a DAF task is equivalent when feedback is a delayed version of the speaker's own speech or a nonspeech sound substituted for the delayed speech. This supports 
the view that the delayed signal is not used to check that the sound was articulated properly, since the nonspeech sound cannot be used for this purpose. Disturbance with both types of feedback is affected by the level of the delayed signal; the louder the signal, the more disturbance is experienced.

\section{GENERAL DISCUSSION}

The experiments demonstrate that the amount of disruption under DAF depends on the level of the signal out of synchrony with speech. Experiment 1 shows that susceptibility to the effects of DAF has nothing to do with inherent differences between subjects in the way in which they use feedback, since subjects can alter their susceptibility by changes they make to the level of their voices. Experiment 2 shows that the out-of-synchrony signal does not have to be speech to produce the sort of disturbance that has previously been interpreted as supporting a process of feedback control in speech production. Susceptibility cannot, then, be due to differences in the way in which different speakers use feedback. Rather, the effects of DAF are one example of disruption to activity that occurs when an event not synchronized with the activity is taking place.

We will now consider how the experimental findings relate to reports that more fluent speakers are less susceptible to the disruptive effects of DAF. If it is assumed that less fluent speakers rely more on feedback to check that speech is articulated properly than do fluent speakers, these reports might offer support for such feedback monitoring being important for speech control. This would contradict the claim made here that the delayed signal is not used for this purpose. However, there are data in two of the studies outlined in the introduction that are consistent with the explanation of susceptibility outlined here. In the developmental study of Siegel et al. (1980), both sound pressure level and time to read a list under different delays were measured. Siegel et al. (1980) measured these dependent variables at two speech rates (nor$\mathrm{mal} /$ fast). At a normal speech rate, the youngest children spoke loudest and were slowest, 8-year-olds were next loudest and took an intermediate length of time, and adults spoke softest and were the fastest. This relation is consistent with the hypothesis that levels across the different age groups determine how disrupted the different speakers were. Unfortunately, this relation does not hold at the speeded rate. This may be because, although it is natural for all groups to speak at a normal rate, it is difficult for children (but not for older groups) to alter their speech rates. Thus, speaking rapidly may differentially affect subjects of different ages, thereby invalidating comparisons across age groups at speaking rates other than the normal rate.

It was noted in the introduction that males are less fluent under DAF than females (Bachrach, 1964). In Bachrach's study, males were not only more susceptible, but also spoke loudest. Thus, again a relationship between voice level and susceptibility occurs across speakers. Bachrach (1964) also noted that females become as susceptible as males if they raise their voice levels. Thus, there are indications that the findings on groups that differ in fluency might also be explained because of differences in voice level rather than a different reliance on feedback.

\section{REFERENCES}

BACHRACH, D. L. (1964). Sex differences in reactions to delayed auditory feedback. Perceptual and Motor Skills, 19, 81-82.

BLACK, J. W. (1951). The effect of delayed sidetone upon vocal rate and intensity. Journal of Speech and Hearing Disorders, 16, 56-60.

BuRKE, D. D. (1975). Susceptibility to delayed auditory feedback and dependence on auditory or oral sensory feedback. Journal of Communication Disorders, 8, 75-96.

Butler, R. A., \& Galloway, F. T. (1957). Factoral analysis of the delayed speech feedback phenomenon. Journal of the Acoustical Society of America, 29, 632-635.

FaIRBANKS, G. (1955). Selected vocal effects of delayed auditory feedback. Journal of Speech and Hearing Disorders, 20, 333-345.

Howell, P., Powell, D. J., \& KHAN, I. (1983). The amplitude contour of the delayed signal and interference in delayed auditory feedback tasks. Journal of Experimental Psychology; Human Perception and Performance, 9, $772-784$.

KorRowBrow, N. (1955). Reaction to stress: A reflection of personality trait organization. Journal of Abnormal and Social Psychology, 51, 464-468.

MACKAY, D. G. (1970). Why does language familiarity influence stuttering under delayed auditory feedback? Perceptual and Motor Skills, 30, 655-669.

RANKIN, R. J., \& BALFREY, W. R. (1966). Impact of delayed auditory on the IPAT 8-Parallel Form Anxiety Scales. Psychological Reports, $18,583-586$.

Siegel, G. M., Fehst, C. A., Garber, S. R., \& Pick, H. L. (1980). Delayed auditory feedback with children. Journal of Speech and Hearing Research, 23, 802-813.

YATES, A. J. (1963). Delayed auditory feedback. Psychological Bulletin, 60, 213-232.

\section{NOTES}

1. It does not follow from the Howell et al. (1983) account of DAF that the delay causing most disruption corresponds to the length of the programming unit in speech production.

2. Some alteration to the measured sound pressure level would occur if subjects moved their heads. No head movements were noted, however, and certainly none occurred that were correlated with feedback delay.

(Manuscript received October 10, 1983; revision accepted for publication July 24,1984 .) 